\title{
Illuminating the Inner Retina of Vertebrates: Multiple Opsins and Non-Visual Photoreceptors
}

\author{
Mario E. Guido \\ CIQUIBIC-CONICET, Departamento de Química Biológica "Ranwel Caputto", Facultad de Ciencias Químicas, Universidad Nacional de \\ Córdoba, Córdoba, Argentina.
}

E-mail: mguido@fcq.unc.edu.ar

"Just as our ears provide us with the sense of balance and hearing, each of our eyes is essentially two organs in one"

Ignacio Provencio, Scientific American 2011

\begin{abstract}
Throughout evolution, the need to detect light has generated highly specialized photoreceptor cells that in vertebrates are mainly located in the retina. The most studied photodetectors within these cells are the visual photoreceptors "cones and rods" responsible for day and night vision, respectively. These cells contain photosensitive molecules consisting of a protein part called "opsin" that binds a chromophore derived from vitamin A, retinaldehyde, capable of photoisomerizing from 11-cis retinal to all-trans retinal form, and triggering the light responses that lead to vision. However,

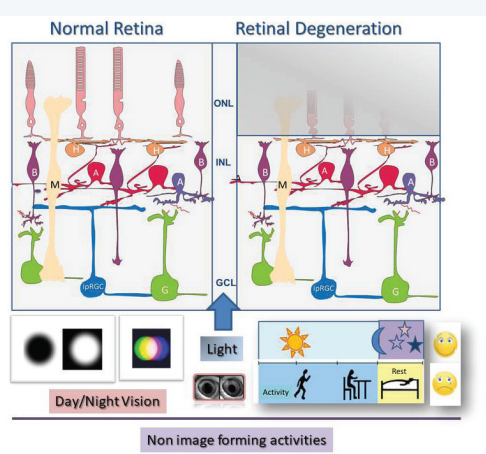
other cells of the inner retina of vertebrates [retinal ganglion cells (RGCs), horizontal cells (HCs), and Muller's glial cells] are currently known to express non-visual photopigments such as melanopsin (Opn4), encephalopsin (Opn3) and neuropsin (Opn5), which would be involved in diverse functions not associated with imaging. Melanopsin is the most widely studied of them, it is expressed in intrinsically photosensitive RGCs (ipRGCs) and HCs of the chicken retina and participates in setting the biological clock, the pupillary light reflex, and presumably in other reflex and subconscious functions, in addition to the lateral interaction between visual photoreceptors and HCs. It is noteworthy that these non-visual photopigments (Opn3, Opn4 and Opn5) respond to blue and/or near violet region light. This particular photosensitivity may provide individuals with a broader spectrum of response to light stimulation within the visible beyond the scope of the visual photoreceptors, regulating an important number of functions not yet completely identified. We can conclude that "a constellation of cells and photoreceptor molecules are present in the inner retina of vertebrates, and from very early stages of development, even before any sign of vision may occur."
\end{abstract}

\section{Keywords:}

retina, non-visual phototransduction, opsins, blue light, non-image forming activities, ganglion cells, horizontal cells, photoreceptors

\section{Introduction}

Light is itself an essential source of energy and life on the planet. Many living organisms have developed specific molecules for the detection of light, known as "photopigments" mainly in the visible spectrum. They are located in specialized cells called "photoreceptor cells" placed in the retina of all vertebrates including man (Fig. 1), and in other photosensitive structures in non-mammalian vertebrates such as the pineal gland and nuclei of the deep brain. 
The detection of light by such photopigments triggers both the functions associated with vision that involve image formation, and a significant number of reflex and subconscious activities, not associated with imaging. These functions include phototaxis, setting of biological clock by light, pupil constriction, sleep, light inhibition of the hormone "melatonin" in the pineal gland (night signal) and photophobia, among others. Likewise, prolonged deficits or persistent alterations in the lighting environmental conditions due to seasonal changes (short days with low light intensity and long nights), industrialized modern life with continuous artificial lighting day and night, night exposure to daylight tablets, notebooks, cell phones, LED screens and televisions, night work-shifts, or trans-meridian flights, cause marked alterations in physiology and behavior. These include sleep disturbances, the desynchronization of daily behavioral and hormonal rhythms, jet lag, seasonal depression, etc. The latter, called SAD (Seasonal Affective Disorder), are precisely treated with phototherapy by exposure to bright blue light at day to counteract the poor ambient light on short winter days.

Through the perception of light, most living beings can adequately visualize the surrounding environment, as well as perceive the marked changes in light intensity, spectral composition, duration and contrasts that occur throughout the day and night. In animals, such light perception constitutes the primary event that triggers more complex processes leading to the formation of images and non-visual functions. For this, photopigments capture photons and convert them within photoreceptor cells into chemical information and electrical signals. In higher animals, these signals propagate through nerve impulses from the retina to different brain regions. Photopigments called "opsins" are proteins that bind a vitamin A derivative, the retinaldehyde, as a chromophore, able to photoisomerize, making them sensitive to light. Opsins are located in the membranes of specialized cells: "the photoreceptors". Visual photoreceptors are grouped together forming structures of varying complexity: the eyes (Fig. 1).
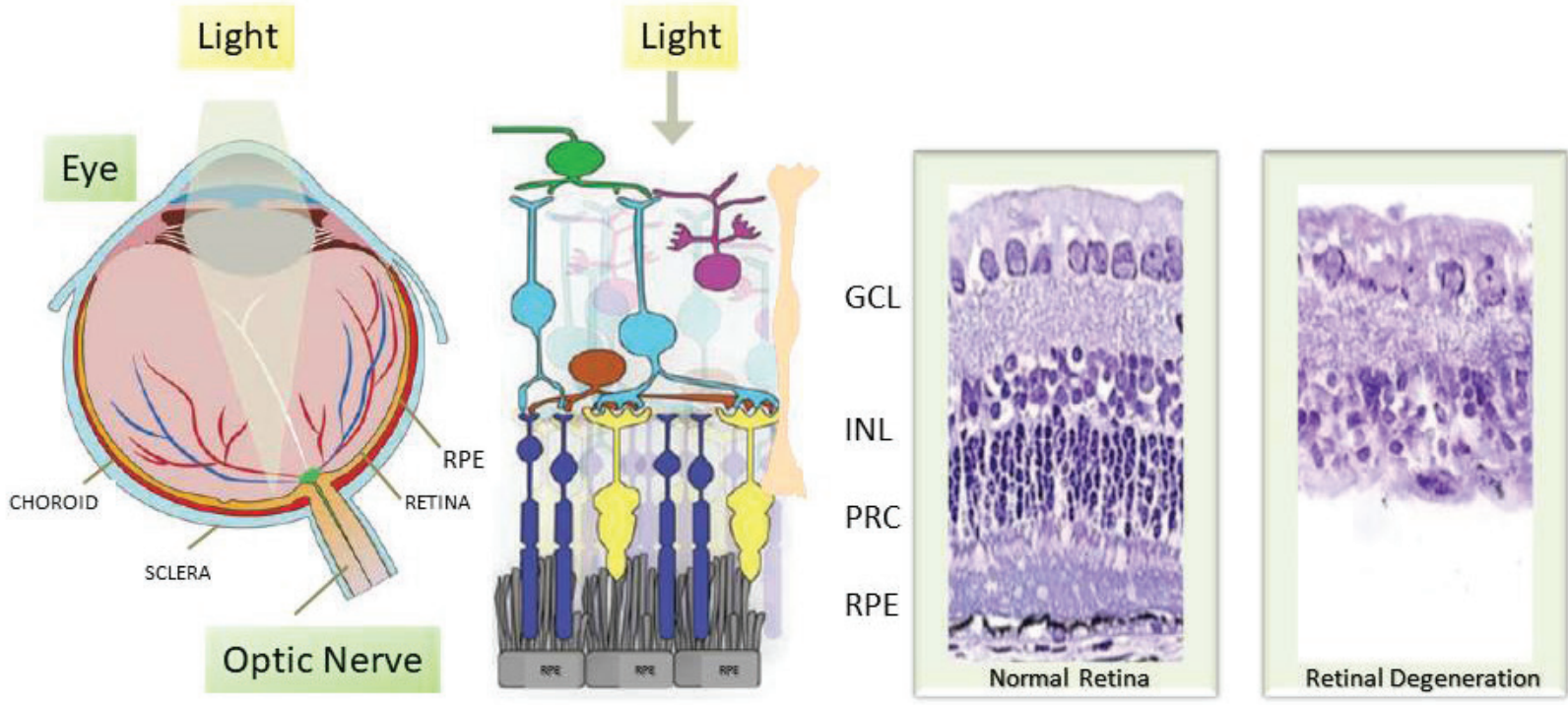

Figure 1. Illustration of the Eye and Retina of vertebrates. Left panels: The retina lies at the back of the eye, it is a multilayered structure that is in contact with the pigment epithelium (RPE) and is made up of Photoreceptor Cells (PRC): cones (yellow) and rods (blue) with external and inner segments and their nuclei forming the outer nuclear layer (ONL). This layer synapses with the outer plexiform layer (OPL) of the inner nuclear layer cells (INL) made up of interneurons: Horizontal Cells (brown), Amacrine (violet) and Bipolar (light blue) whose processes form the inner plexiform layer (IPL) that contacts the Ganglion Cells (GCs, green) of the ganglion cell layer (GCL) whose axons form the optic nerve (green) and project to the brain. Light first strikes these GCs, which in turn contact the feet of Muller Glial Cells (pink) whose body extends throughout the entire inner retina. Right panels: Histological staining of the retina from a healthy control and from a blind animal suffering retinal degeneration and lacking the photoreceptor cell layer (PRC), whereas the inner retina remains viable (INL and GCL).

From an evolutionary point of view, the vertebrate eye has a very important morphological and functional complexity, comprising the eye chamber, which has a set of systems that collects light and projects it onto the retina, located at the back of the eye (Fig. 1). The retina is a multilayered structure that is part of the central nervous system and consists of three neuronal layers (layers of neuron nuclei) and two layers of processes (plexiform layers). The retinal photoreceptor cells 
(PRCs), so-called "visual photoreceptors", are the rods and cones, which are responsible for daytime (color) and night (black and white) vision, respectively. The PRCs are in contact with the retinal pigment epithelium (RPE) that prevents the diffusion of light, and that are in charge of recycling the visual chromophore through the process called "visual cycle". The interneurons (horizontal cells or HCs, bipolar and amacrine cells), through synapses, connect the PRCs with the retinal ganglion cells (RGCs) whose axons form the optic nerve that conducts nerve information to different areas of the brain involved in vision (visual circuit) and / or through an independent "non-visual" circuit, responsible for the control of subconscious and reflex functions (Fig. 2).

\section{Visual \& Non Visual Circuits}

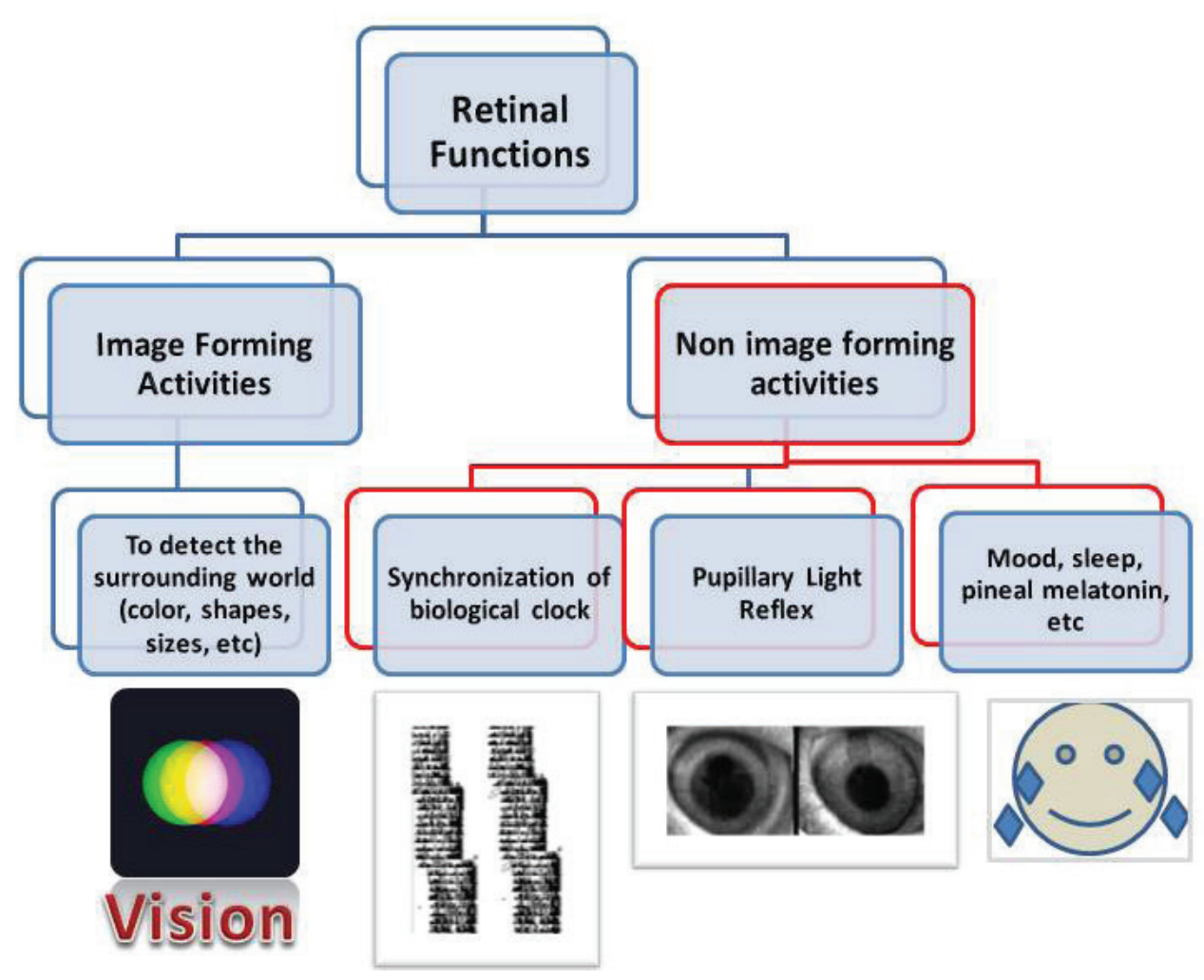

Figure 2. Visual and Non-visual Circuits. Retinal functions. Image forming and Non-image forming tasks. The Visual Circuit (blue) projects from the retina to the brain (visual cortex and other brain areas) and is responsible for coordinating the image-forming activities that lead to vision and allow us to visualize the surrounding environment. The non-visual circuit (red) projects from the retina to various brain areas regulating the physiology and behavior of vertebrates mainly related to subconscious and reflex functions. In non-mammalian vertebrates, light can be detected by the retina, the pineal gland, and deep brain photoreceptors. In GUCY1 * chickens that are blind from birth, light still regulates the daily timing of feeding rhythms (double plot actogram shown below) and the pupillary light reflex (image of a GUCY1 * chicken pupil before and after light stimulation). Light is detected through retinal ganglion cells (RGCs), intrinsically photosensitive (ipRGCs) that project to the brain areas forming a non-visual circuit (red), independent of image-forming activities. This circuit regulates synchronization of daily rhythms by light, pupillary light reflexes, photic inhibition of pineal melatonin, mood, sleep, and other non-visual functions. See text for further details.

At the beginning of the last century, an advanced student at Harvard, Clyde Keeler (1927) observed that his blind pet experienced constriction of the pupils when exposed to light. This intriguing observation induced the question whether it is possible to perceive light even in the absence of vision. These and closely related questions have motivated numerous researchers around the world throughout the last decades and have stimulated them to answer these questions. 


\section{Unknown functions for photosensitive cells of the inner retina}

Only at the beginning of the $21^{\text {st }}$ century, two fundamental studies shed light on the existence of a circuit different from that related to image-formation, which would be responsible for such photosensitivity. One of these investigations was carried out by Ignacio Provencio and colleagues (2000) who identified a photopigment called melanopsin (Mel or Opn4) (Fig. 3) expressed in mammals in a subpopulation of RGCs. The other by David Berson together with Samer Hattar and collaborators (2002) who described that a subpopulation of RGCs responded directly to light, calling them intrinsically photosensitive RGCs (ipRGC). The peculiarity of these cells is that they project to the anterior hypothalamus, specifically to a small nucleus called the suprachiasmatic nucleus (SCN) and to other "brain" regions. The SCN constitutes the master clock of mammals and is responsible for the temporal control of physiological and behavioral rhythms (Fig. 2). Furthermore, these ipRGCs express the melanopsin photopigment and are also present in non-mammalian vertebrates such as birds, fish and amphibians. Within these vertebrates, the chicken has been the working animal model in our laboratory and we currently know that these birds also contain photosensitive cells in their inner retinas, forming a neural network that feeds an independent non-visual circuit. Specifically, these ipRGCs would be responsible, through projections to specific areas of the brain, of daily synchronizing the biological clock to external lighting conditions, of controlling the pupillary reflex, and of regulating by light other processes not associated with imaging (review: Guido et al., 2010) (Fig. 2).

Against this background, a fundamental question arises about how these ipRGCs act and transmit information to the brain. To answer this question, we had to isolate the ipRGCs, keep them in culture and study their responses to light, at the biochemical and molecular levels, as well analyze the nature of the potential phototransduction cascade. Our group first described the photocascade that takes place in any vertebrate ipRGCs through studies with primary cultures of chicken RGCs at early embryonic stages, in which the only differentiated retinal cells are these RGCs. We applied molecular, biochemical and pharmacological tools (Contin et al., 2006; 2010). From these studies, we observed that these photosensitive cells act through a photocascade that involves retinaldehyde as the chromophore, a Gq protein as a transducin, and a phospholipase $\mathrm{C}$, - an enzyme that hydrolyzes phosphoinositides - as an effector enzyme, ultimately causing mobilization of intracellular calcium, and the activation of TRP-like transient potential receptors (Contin et al., 2006; 2010; Diaz et al 2014; 2017) (Fig. 4). The mobilization of intracellular calcium induces the depolarization of the cell and the light-triggered process is highly similar in its biochemical nature to the phototransduction cascade that occurs in invertebrate photoreceptors (rhabdomeric photoreceptors). Furthermore, these ipRGCs would derive from an ancestral photoreceptor common with invertebrate photoreceptors, which has endured throughout evolution coexisting with the rods and cones within the retina, in order to complement the activity of these visual photoreceptors (day and night vision). Likewise, ipRGCs appear very early in the development of vertebrates, before any specific signal of formal vision is detected. The question that then arises is: What would be the function of these early photoreceptor cells? In fact, it is sensing light and darkness at day and night. We currently know that these intrinsically photosensitive cells are responsible for photodetection mainly associated with light control of various non-visual functions. 


\section{VISUAL AND NON VISUAL PHOTOPIGMENTS}

\section{Photoreceptor Cells}
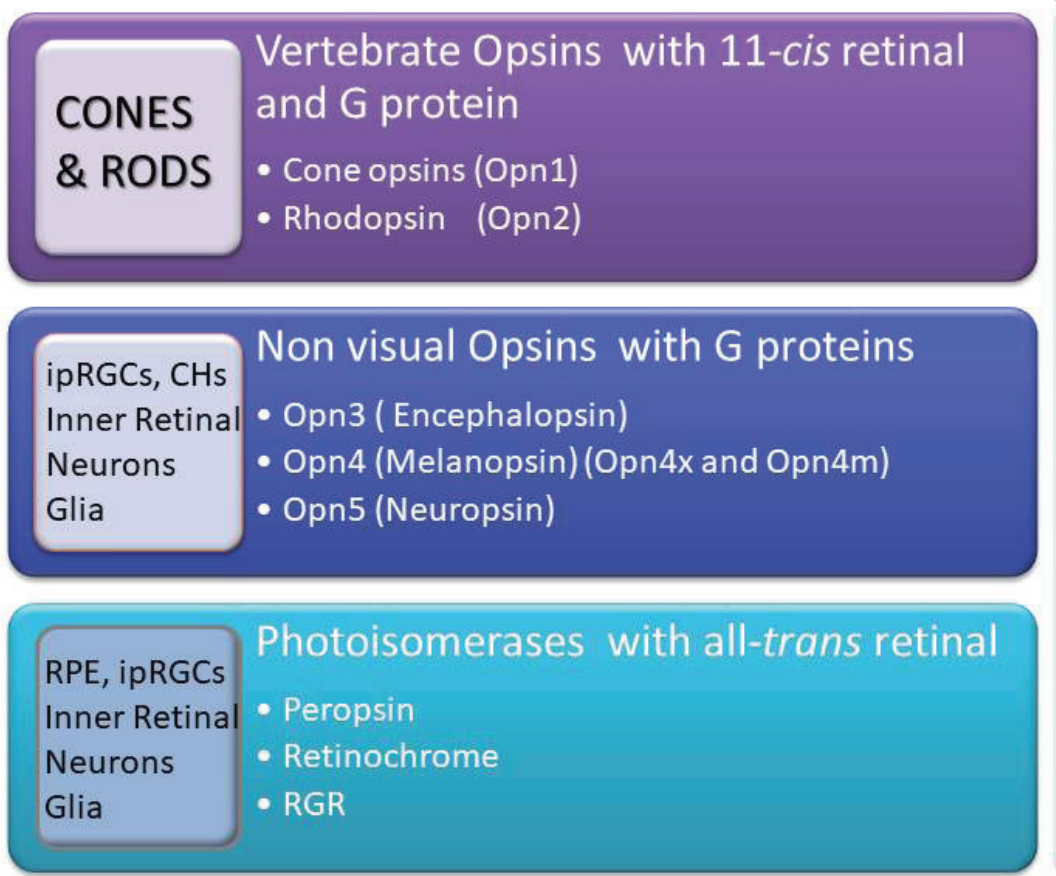

Photosensitivity/

Photocascade

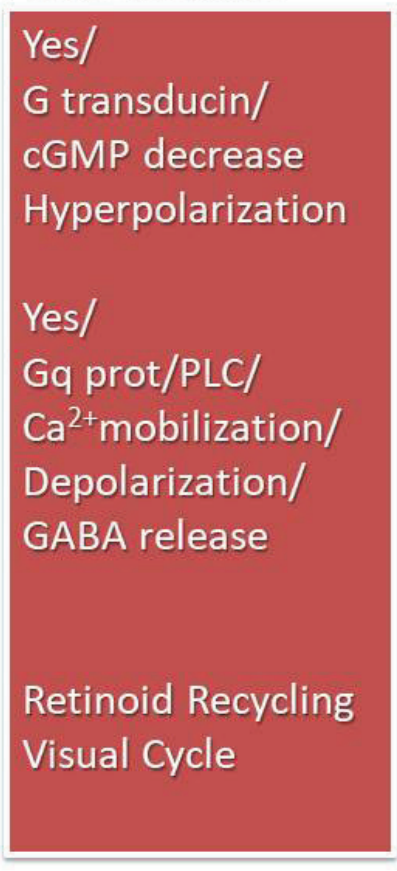

Figure 3. Visual and Non-visual Photopigments and Photoisomerases. Visual Opsins are the visual photopigments that include short, medium and long wavelength opsins (Opn1) and rhodopsin (Opn2) present in the outer segments of cones and rods (visual photoreceptor cells) and are involved in day and night vision, respectively. The chromophore 11-cis retinal (vitamin A derivative) is bound to a lysine residue in the protein part of the photopigment. Upon light stimulation, 11-cis retinal is photoisomerized to all-trans retinal. Opsins are coupled to a $\mathrm{G}$ protein that acts as the light transducer.

Non-Visual Opsins: include opsins that use retinal as a chromophore and bind to a G protein. These opsins include: Opn3 (encephapsin), Opn4 (melanopsin), and Opn5 (neuropsin). The peculiarity of these opsins is that they respond to blue (Opn3 and Opn4) and UV (Opn5) light and are involved in the regulation of various light-driven non-visual functions. Melanopsin: Throughout evolution, vertebrates have conserved 2 Opn4 genes (Opn4m and Opn4x). Opn4x has been lost in mammals along with other opsins, likely during the mammalian entrance into the nocturnal phase. Non-mammalian vertebrates (birds, fish, etc.) possess both Opn4 genes (Opn4m and Opn4x), which encode for the corresponding proteins and isoforms differentially expressed in ipRGCs and HCs of the inner retina. The phototransduction cascade triggered by Opn4 activation, involves the Gq protein, phospholipase $\mathrm{C}$ (PLC) activation, $\mathrm{Ca}^{2+}$ mobilization, and membrane depolarization with GABA release in the inner retina. Opn3 and Opn5 are expressed in neurons of the inner retina and in Müller glial cells through cascades and functions not yet fully elucidated. The third group involves Photoisomerases that bind all-trans retinal: Peropsin, Retinochrome and RGR. They are expressed in the retina of vertebrates, mainly in the retinal pigment epithelium (RPE), inner retinal cells and Muller glial cells, and involved in the recycling of the chromophore required to constitute an active photopigment.

\section{Various photoreceptors/photopigments coexist in the vertebrate eye}

It is really remarkable that, although there is a variety of eye types with greater or lesser complexity in nature, only a very limited number of photoreceptor cell types are observed: the photoreceptors of invertebrates called "rhabdomerics" and the ciliated photoreceptors of vertebrates (PRCs cones and rods, Fig. 1), and photoreceptors of the pineal gland in non-mammalian vertebrates. Rhabdomeric photoreceptors present a membrane arrangement organized in microvilli where the photopigments are concentrated, whereas the ciliated photoreceptors present the cilium folded back on itself, forming saccules in the external segment that contains the opsins. In both types of photoreceptors, opsins are covalently bound to the vitamin A-derived chromophore, the retinal. However, and beyond the fact that the different types of opsins have a certain homology, they use different nervous transduction systems with opposite electrochemical signals. Rhabdomeric photoreceptors are depolarized by light (becoming more positive inside), whereas ciliated photoreceptors 
are hyperpolarized by light (becoming less positive inside). In invertebrates, rhabdomeric photoreceptors, associated with pigment cells, form the eyes, whereas in vertebrates they would have transformed into RGCs (ipRGCs) and other cells of the inner retina such as amacrine and horizontal cells (HCs). These retinal neurons are evolutionarily sister cells with RGCs by sharing specification factors and also retaining intrinsic photosensitivity as described above (Fig. 3). On the other hand, ciliated photoreceptors evolved into photoreceptors of the pineal organ of the brain and in the retina into rods and cones. The cones are sensitive to intense light and have opsins that respond to different wavelengths (short, medium and long; day vision, color), whereas the rods are sensitive to low-intensity light (night vision).

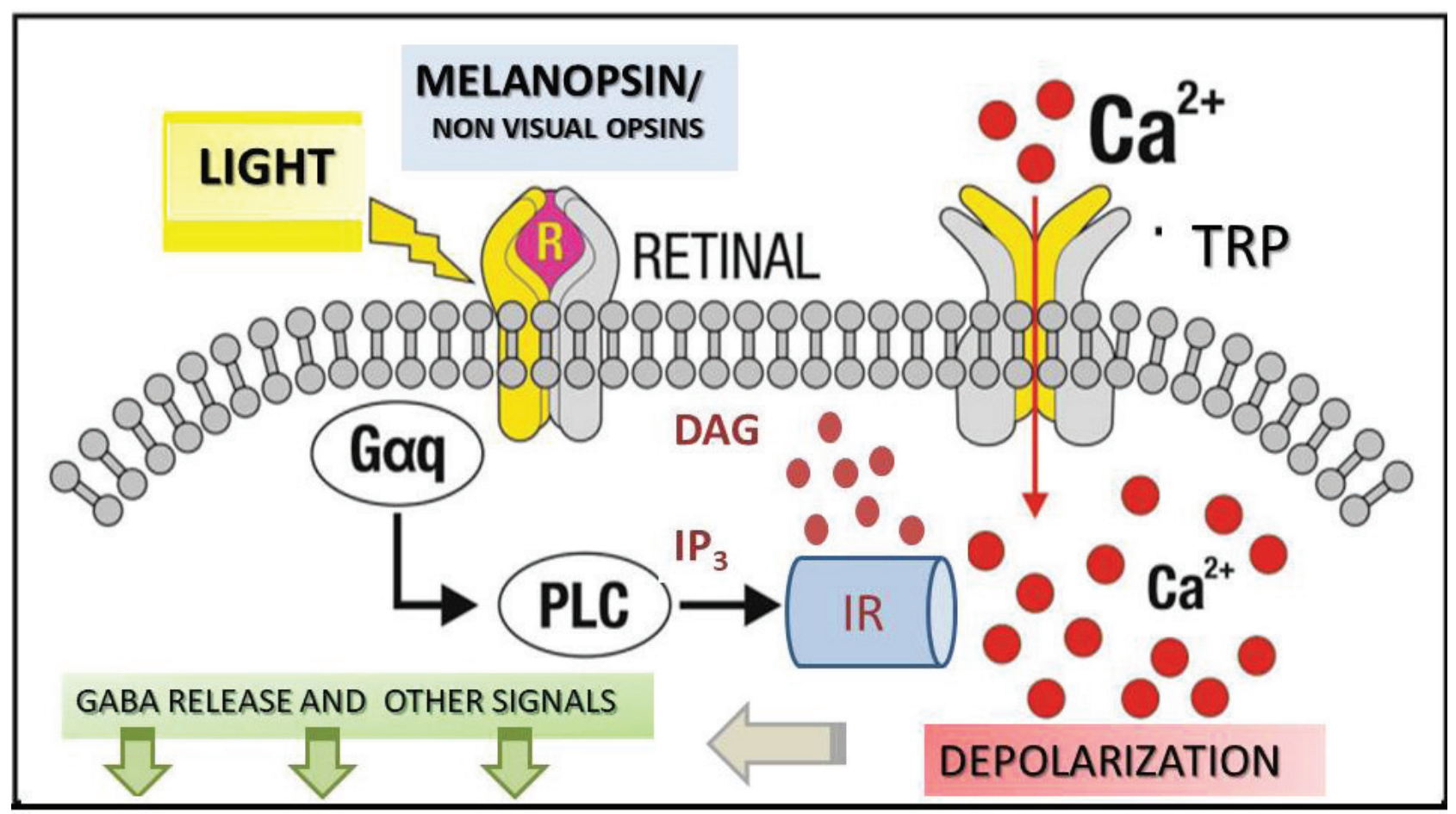

Figure 4. Phototransduction cascade of non-visual photoreceptors of vertebrates. This photocascade operates in intrinsically photosensitive ganglion cells (ipRGCs) and horizontal cells (HCs) expressing melanopsin proteins (Opn4m and Opn4x). Once a photon strikes the melanopsin or another non-visual photopigment, the retinal chromophore isomerizes from 11-cis retinal to all-trans retinal. This photoisomerization activates a Gq protein that acts as a light signal transducer activating an effector protein, the enzyme phospholipase C (PLC). In turn, PLC hydrolyzes the phosphoinositides (PIP ${ }_{2}$ ), minor lipids of the membranes, producing intracellular lipid mediators (inositol-triphosphates $\mathrm{IP}_{3}$ and diacylglycerol DAG) with the consequent $\mathrm{Ca}^{2+}$ mobilization inside the cell through inner reservoirs (IR) or transient potential channels located in the membrane (TRP) (Contin et al., 2006; 2010, Diaz et al., 2014). The increase of $\mathrm{Ca}^{2+}$ inside the cell produces the depolarization of the membrane, making it more positive inside with the consequent release of the GABA neurotransmitter in HCs (Morera et al., 2016) or other neurochemical signals.

In the vertebrate retina, photoreceptors also differ in the functions they regulate since PRCs are responsible for the processes required directly for vision, and associated with imaging, whereas ipRGCs regulate reflex and subconscious functions (Fig. 2). Based on the presence of the melanopsin photopigment, on the biochemical characteristics of the photocascade triggered in response to light, and on a group of transcription and specification factors, phylogenetically conserved and expressed by these cells (Pax6, Ath5, Brn3 and BarH genes), it could be concluded that these inner retina cells of vertebrates (ipRGCs, HCs and amacrine) share a common evolutionary ancestor with the photoreceptors of invertebrates. Furthermore, our group also recently demonstrated that in the chicken embryonic retina, HCs that express a particular isoform of the melanopsin photopigment (mel x) exhibit photosensitivity to blue light causing cell depolarization with calcium mobilization and GABA release (Morera et al., 2016). It is noteworthy that these HCs are in direct contact with the visual photoreceptor cells "cones and rods" through the outer process / plexiform layer, and could have the dual role of regulating lateral interaction in response to blue light or cooperate with the ipRGCs circuit to control lighttriggered non-visual functions. 


\section{A variety of new photopigments illuminate the inner retina of vertebrates}

The first evidence supporting the concept that the melanopsin photopigment is responsible for the aforementioned photosensitivity came from a series of pioneering studies showing that immortalized non-retinal cells became photosensitive after induction of melanopsin expression (heterologous expression), whereas on the other hand, genetically engineered mice not expressing melanopsin and lacking functional cones and rods, completely fail to detect all types of light stimulation (reviewed in Guido et al, 2010, and Diaz et al., 2015).

The photopigment melanopsin was initially described by Ignacio Provencio in the frog (Xenopus) and later it was found that it is also expressed in the brain, iris and retinal cells of most vertebrates (Provencio et al., 2000). There are at least 2 genes that encoded for melanopsin: Mel-x (Opn4x) and Mel-m (Opn4m), with homologies to the Xenopus (Opn4x) or mammalian (Opn4m) genes, respectively (Bellingham et al., 2006) (Fig. 3). It is noteworthy that Mel-m is only expressed in mammals, whereas in birds, amphibians and fish both Mel-m and Mel-x are expressed. The loss of Mel-x by mammals could be due to the entry of mammals into the night niche, which produced a significant reduction in photosensitivity, so that as compared to marsupials, mammals suffered the loss of the genes of Mel-x and of some visual opsins. Although some of the visual opsins were later recovered by gene duplication it was not the case of Mel-x. On the other hand, in birds and other non-mammalian vertebrates, more complex photodetection systems were preserved during evolution including the pineal organ and deep brain photoreceptors, responsible for measuring day length and involved in processes such as seasonal migration and reproduction (Valdez et al., 2009; 2013; reviewed in Guido et al., 2010).

In birds, the presence of messenger RNAs and proteins has been reported for both Mel-x and Mel-m photopigments. The different melanopsin proteins and their isoforms display sensitivity to blue light close to $480 \mathrm{~nm}$, are expressed in the retina and are confined in a first stage of development to the RGCs, their axonal fibers and along the optic nerve that connects the retina with the brain (Verra et al., 2011). All these findings were observed at stages at which retino-tectum projections are formed. At later and postnatal embryonic stages, a marked spatial separation is observed between both proteins, whereas Mel-m remains confined to a subpopulation of RGCs, Mel-x is also expressed very strongly in other cells of the inner retina, reaching maximum expression in the outer plexiform layer that contacts rods and cones and comprises HCs. Such HCs reach their differentiation and final destination within the retina, coinciding with the robust expression of Mel-x and its colocalization with the transcription factor Prox1, specific marker of HCs. Indeed, the opsin Mel-x is expressed in sister cells of the ipRGCs, the HCs, which have also retained the intrinsic photosensitivity as we recently demonstrated (Morera et al., 2016).

\section{Non-visual opsins and photoisomerases present in the inner retina of vertebrates}

In addition to the melanopsin that is expressed in ipRGCs and HCs, other photopigments have been described in the inner retina of vertebrates; they are the opsins Opn3 (called encephalopsin) and Opn5 (called neuropsin), and the photoisomerase RGR (Retinal G protein coupled Receptor), whose expression we have found in rat and chicken retina (Nieto et al., 2011; Diaz et al., 2017; Rios et al., 2019) (Fig. 3). The Opn3 and Opn5 photopigments belong to the family of G protein-coupled opsins that bind retinaldehyde as a chromophore, and respond to blue and UV light, respectively. The generic name of both opsins is due to the fact that they were first found expressed in the brain, but today it is known that they are expressed in the vertebrate retina, and in extra-retinal tissues, such as the skin, with potential functions not yet fully characterized. In chicken, both opsins are expressed in nerve cells of the embryonic inner retina and in Müller glial cells (Rios et al., 2019). Furthermore, primary cultures enriched in Müller's glial cells that express these photopigments respond to blue light of high intensity and prolonged duration ( $>20$ seconds), mobilizing intracellular calcium, through a cascade not completely characterized even from the biochemical point of view (Rios et al., 2019) (Fig. 3). These novel findings suggest the possibility that these retinal glial cells are detecting long-term light (prolonged stimuli) and generating responses that modulate the interaction between cells or between glia and neurons.

Likewise, other studies carried out in our laboratory showed that RGR photoisomerase is expressed in early stages of embryonic development in neurons and glia, and especially in melanopsin (+) ipRGCs (Diaz et al., 2017). This photoisomerase would be essential to regulate by light the endogenous content of retinoids (retinals, retinols and retinyl esters) required by the inner retina to provide the photopigments with enough active chromophore needed for the photic responses.

\section{From the retina to the brain. Retinal photoreceptor cells and visual circuits. Experi- mental models for the study of non-visual photoperception.}

The vertebrate eye always acts as a sensor for ambient lighting conditions even in the absence of vision. In any case, and even in the face of these conditions, the retina is the gateway for light information that regulates different non-visual 
functions that synchronize the physiology and behavior of organisms with the external environment (day-night / light cycles- darkness) (review in Guido et al., 2010) (Fig. 1 and 2). Indeed, the presence of a different group of photoreceptors housed in the inner retina of vertebrates and containing non-visual photopigments of maximum absorbance in the blue region, has given vertebrates the evolutionary advantage of higher photosensitivity in a spectral region wider than that covered by rods and cones, and has enabled them to regulate other functions, not only those associated with imaging.

In humans, even suffering certain types of blindness with progressive retinal degeneration, reflection of their pupils to light stimulation or acute inhibition by light of the nocturnal pineal melatonin production can be observed. This strongly indicates that a light detection system that functions to regulate such non-visual functions by light still remains in the retina of these individuals. These observations, transferred to animal models of mammals with retinal degeneration, mainly in mice, with the consequent loss of their PRCs, show the existence of a different population of non-canonical retinal photoreceptors - the ipRGCs - responsible for regulating functions not associated with imaging. To perform studies on non-mammalian vertebrates, we used wild-type Rhode Island chickens as healthy controls together with GUCY1* chickens suffering a retinopathy similar to Leber's congenital amaurosis (LCA) in humans. These chickens suffer blindness from birth and have their PRCs (cones and rods) non-functional and subject to progressive degeneration (Valdez et al., 2009; 2013). However, these animals maintain functional the rest of their retina, constituting an experimental model of important utility to evaluate the persistence of photoperception under pathological conditions, the functioning of RGCs and other cells of the inner retina (neuronal or glial types), the participation of them in non-visual photoreception mechanisms, and the type of photocascade and mechanisms that take place in response to light. GUCY 1 * chickens carry a mutation in the guanylate cyclase enzyme involved in phototransduction that inactivates this enzyme, with the consequent lack of production of cyclic GMP, the second messenger essential in the activation of cationic channels that generate the dark current in the absence of light in visual photoreceptors (cones and rods).

After developing a colony of this LCA model in Córdoba, in a first stage we proceeded to characterize these animals with histological and electroretinographic studies. These animals were clinically blind, with their PRCs cones and rods completely non-functional. During the first weeks of life, the retinas of GUCY ${ }^{*}$ chickens appear morphologically normal. However, after ca. 3 months of life, the PRC layer shows signs of degeneration, whereas the rest of the inner retina (inner nuclear layer and RGCs) remains intact (Fig. 1, histological staining in right panel). On the other hand, these animals do not show any electrical activity in response to light of diverse intensities and wavelengths, at any age (Valdez et al., 2009). In a second stage we proceeded to study possible responses to light, evaluating behavioral and physiological parameters that were light regulated. To this end, and since the chickens have daytime habits, we investigated the light synchronization of the daily feeding rhythms and the pupillary constriction reflex. Every time the animals are fed, a signal is recorded through a feeding system with an infrared detector, which generates 24-hour recordings collected for weeks and months so-called "actograms". These "feeding rhythms" recordings were utilized with both GUCY1* chickens and their healthy controls after exposure to different lighting conditions (see bottom panel Fig. 2). The blind animals adjusted their daily feeding rhythms with precision to the different imposed lighting phases and to light-darkness (LD) patterns with 4 hours advance or delay, in the same way as the control animals. These daily rhythms persisted in the blind even with occlusion of their extra-retinal photoreceptors located in the pineal gland and deep brain, and / or after the surgical removal of the pineal gland (pinealectomy) (Valdez et al., 2009, 2013). Even in blindness, these animals perceive light that accurately synchronizes their rhythms of feeding activity to the imposed cycles or enter "free run" (endogenous rhythm) in constant conditions of darkness (DD) or light (LL ) with periods close to $24 \mathrm{~h}$. However, these responses are lost with enucleation (removal of eye cells), indicating that cells of the inner retina (ipRGCs) are responsible for this light regulation.

By measuring pupil constriction in $\mathrm{GUCY} 1^{*}$, in response to different light intensities and wavelengths within the visible spectrum, another light-controlled function can be addressed, and by performing a sensitivity spectrum, we were able to investigate what type of photopigment is controlling such a function. From a dose-response curve, we determined the sensitivity spectrum (responses to light of certain wavelengths) for the consensual pupillary reflex (one eye exposed to lights and recorded in the other). Indeed, the GUCY1* chickens maintain the consensual pupillary light reflexes as well as the control animals, so that the contraction of the pupil persists in response to light stimulation, even in the absence of classic PRCs (Valdez et al., 2009; 2011) (see bottom panel Fig. 2). The responses obtained indicated that pupillary light reflexes are mediated by an opsin based on vitamin A2, with maximum absorbance at $484 \mathrm{~nm}$, coinciding with the the photopigment melanopsin absorption spectrum (Valdez et al., 2009). Our studies also showed that this photopigment is expressed in the RCCs of healthy and blind chickens (Valdez et al., 2009, Verra et al., 2011). The pupillary reflex is mediated by a subpopulation of RGCs projecting to the pretectal region (OPN) and the Edingher-Westpal nucleus (EW) that bilaterally innervate the ciliary ganglion (CG), producing constriction of the pupil of both eyes (illuminated and contralateral) and indicating the integrity and functionality of the whole circuit (Fig. 2). From these series of studies, it is concluded that GUCY1* chickens, despite clinical blindness, perceive light through the inner retina and their ipRGCs. This perception allows them to regulate different non-visual activities: the setting of the daily rhythms, and the pupillary light reflex. Furthermore, subsequent studies showed that the maximum pupillary constriction is observed during the day and with exposure to blue light (Valdez et al., 2015). 


\section{Conclusions}

From this set of studies carried out with cell cultures and "in vivo" experiments, we can conclude that:

- a constellation of cells and photoactive molecules are present in the inner retina of vertebrates and very early in embryonic development, even before there are concrete signs of the functioning of the vision processes. This happens in different populations of retinal cells that have the ability to respond to light, mainly in the blue spectral region, and complementing the visual function associated with imaging. These populations are: i- Isolated chicken RGRs, characterized and maintained in culture, at very early stages of development. These cells are intrinsically sensitive to light through a transduction cascade with depolarization of the membrane and mobilization of calcium (Contin et al., 2006; 2010; Diaz et al., 2014). These cells express Mel-x and Mel-m as well as the RGR photoisomerase that allows them to recycle the chromophore in light (Contin et al., 2006; 2010; Verra et al., 2011; Diaz et al., 2014; 2017) (Fig. 3).

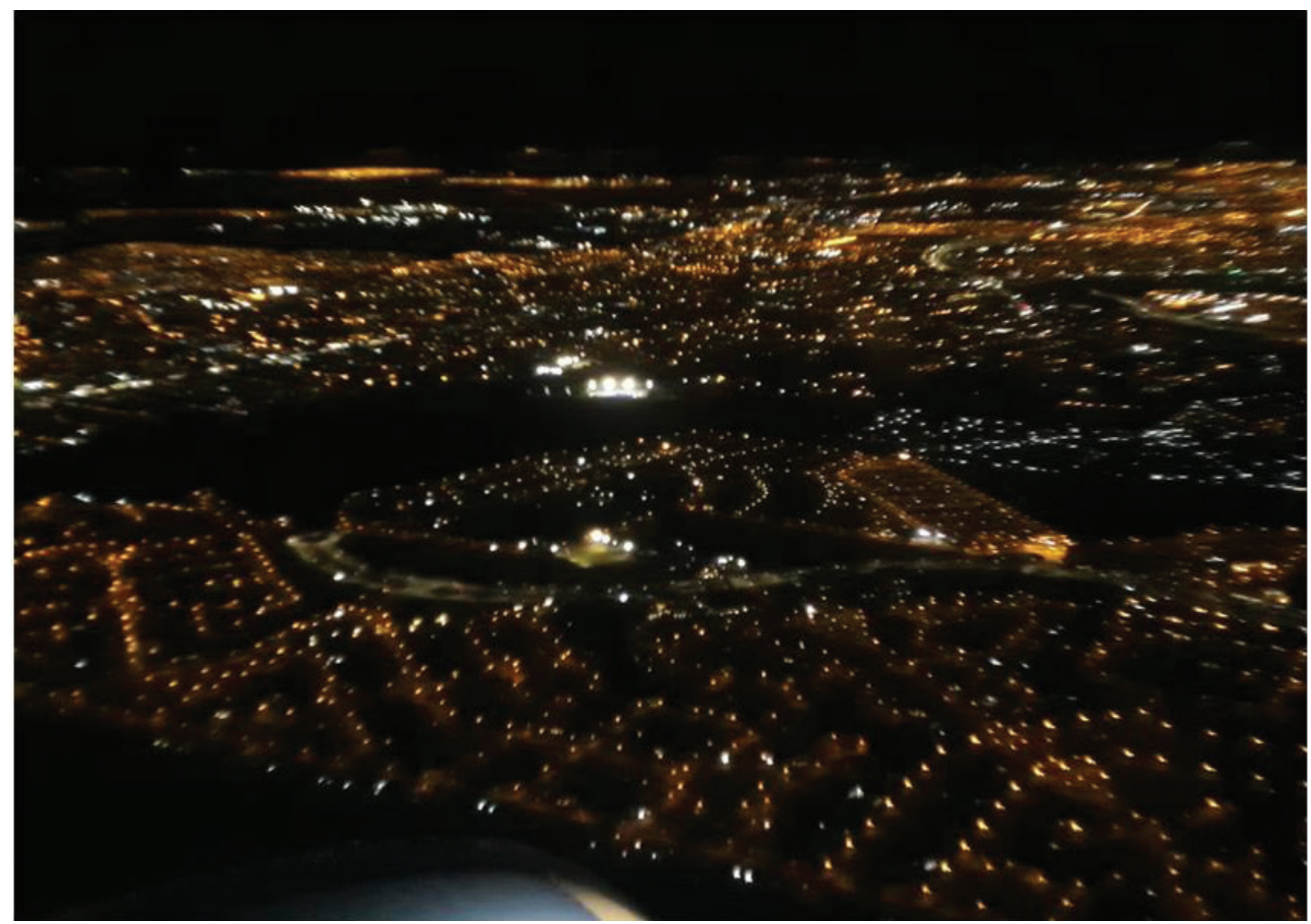

Aerial view photography of Córdoba International Airport area (Córdoba, Argentina) that somehow resembles the multilayer organization of the vertebrate retina and its illuminated visual/non-visual circuits.

ii- The HCs expressing Mel-x that respond to blue light through a photocascade that involves calcium mobilization and release of the GABA neurotransmitter (Fig. 4). These HCs probably act in processes of lateral interaction in contact with cones and rods, and/or cooperating with the ipRGCs in non-visual functions (Morera et al., 2016).

iii- Muller's glial cells and other cells, which express Opn3 and Opn5, respond to longer-time light stimuli, mainly blue light, mobilizing intracellular calcium through a mechanism not yet elucidated (Rios et al., 2019), and collaborating potentially in processes related to neuron-glia or glia-glia interaction under prolongued light stimuli (Figs. 3).

- Through "in vivo" studies with the GUCY1* chickens, we observed that: i) although these animals suffer blindness from birth, they perceive light through their inner retina and their ipRGCs, regulating the pupillary reflex and daily feeding activity (Valdez et al., 2009; 2011; 2013) (Fig. 2). Such photoperception persists even under conditions of occlusion or 
loss of extraocular photoreceptors. However, this photosensitivity is lost with enucleation. These findings highlight a non-visual circuit similar to that described in mammals involving cells of the inner retina, specifically the RGCs and their projections to different brain areas. In fact, their projections to the hypothalamic SCN regulate the photic synchronization of behavior, whereas projections to the pretectal region produce the constriction of the pupil of both eyes (illuminated and contralateral). Nevertheless, new evidence obtained in mammals indicates that ipRGCs could also contribute in some way to vision since some RGCs that express melanopsin also project to visual areas, which opens an intriguing panorama of even greater complexity, potential impact and great challenge for their future study.

\section{Acknowledgments}

This work has been supported by the National Agency for Scientific and Technical Promotion (FONCyT) (PICT 2004 Nr 967, PICT 2006 Nr 898, PICT 2010 Nr 647, PICT 2013 Nr 021, PICT 2016 Nr 0187), National Council for Scientific and Technical Research of Argentina (CONICET), Ministry of Science and Technology of Córdoba (MinCyT-Cba), Secretariat of Science and Technology-National University of Córdoba (SeCyT-UNC); Antorchas Foundation, Florencio Fiorini Foundation, CAEN-ISN and John Simon Guggenheim Memorial Foundation (2009). Author is grateful to Ciencia Hoy for the kind authorization to use Figure 1 from Guido ME (2016) Ciencia Hoy 151, 43-46. ISSN 1666-5171.

\section{References}

[1] Bellingham, J.; Chaurasia, S.S.; Melyan, Z.; et al. Evolution of melanopsin photoreceptors: discovery and characterization of a new melanopsin in nonmammalian vertebrates. PloS Biology 2006, 4, e254.

[2] Berson, D.M.; Dunn, F.A.; Takao, M. Phototransduction by retinal ganglion cells that set the circadian clock. Science, 2002, 295, 5557, 1070-1073.

[3] Contin, M.A.; Verra, D.M.: Salvador, G.; Ilincheta, M.; Giusto, N.M.; Guido, M.E. Light-activation of the Phosphoinositide Cycle in Intrinsically Photosensitive Chicken Retinal Ganglion Cells. Invest. Ophthalmol Vis. Sci. 2010, 51, 11, 5491-5558.

[4] Contin, M.A.; Verra, D.M.; Guido, M.E. An invertebrate-like phototransduction cascade mediates light detection in the chicken retinal ganglion cells. The FASEB J. 2006, 20, 14, 2648-50.

[5] Diaz, N. M.; Morera ,L. P.; Verra, D. M.; Contin, M. A.; Guido, M.E. Early appearance of non-visual and circadian markers in the developing inner retinal cells of chicken. BioMed Res. Int. 2014, 1-9, dx.doi.org/10.1155/2014/646847.

[6] Díaz, N. M.; Morera, L. P.; Guido, M.E. Melanopsin and the Non-visual Photochemistry in the Inner Retina of Vertebrates. Photochem. Photobiol. 2015, 92, 1, 29-44. doi: 10.1111/php.12545.

[7] Díaz, N. M.; Morera, L. P.; Tempesti, T.; Guido, M.E. The Visual Cycle in the Inner Retina of Chicken and the involvement of Retinal G-Protein-coupled Receptor (RGR). Mol. Neurobiol. 2016, 1-11, doi:10.1007/s12035-0169830-5.

[8] Guido, M.E.; Garbarino, E.; Contin, M.A; Valdez, D.; Nieto, P.; Verra, D.; Acosta, V.; de Zabalia, N.; Rosenstein, R.E. Inner retinal circadian ccks and non-visual photoreceptors: novel players in the circadian system. Prog. Neurobiol. 2010, 92, 4, 484-504.

[9] Hattar, S.; Liao, H.W.; Takao, M.; Berson, D.M.; Yau, K.W. Melanopsin-containing retinal ganglion cells: architecture, projections, and intrinsic photosensitivity. Science. 2002, 295, 5557, 1065-70.

[10] Keeler, C. E. Iris movements in blind mice. Am. J. Physiol. 1927, 81, 107-112.

[11] Morera, L. P.; Díaz, N. M.; Guido, M.E. Horizontal cells expressing melanopsin x are novel photoreceptors in the avian inner retina. Proc. Natl. Acad. Sci. USA. 2016, 113, 46, 13215-13220.

[12] Nieto, P.S.; Valdez, D.J.; Acosta-Rodriguez, V.A.; Guido M. E. Expression of novel opsins and intrinsic light responses in the mammalian retinal ganglion cell line RGC-5. Presence of Opn5 in the rat retina. PLoS One, 2011, 6, $10, \mathrm{e} 26417$. ISSN 1932-6203.

[13] Provencio, I. Hidden Organ in Our Eyes Found to Control Circadian Rhythms and Emotions. Scientific American 2011, 304, 5, 54-59. 
[14] Provencio, I.; Rodriguez, I.R.; Jiang, G.; Hayes, W.P.; Moreira, E.F.; Rollag, M.D. A novel human opsin in the inner retina. J Neurosci, 2000, 20, 2, 600-605.

[15] Rios, M.N.; Marchese, N.A.; Guido, M.E. Expression of non-visual opsins Opn3 and Opn5 in the deveping inner retinal cells of birds. Light-responses in Muller glial cells. Front. Cellular Neurosci. 2019, doi: 10.3389/fncel.2019.00376

[16] Tessier-Lavigne M. Phototransduction and information processing in the Retina. In Principles of Neural Science, 3rd edn (Edited by E. R. Kandel, J. H. Schwartz and T. M. Jessell), 1991, 401-418. Appleton \& Lange, Norwalk.

[17] Valdez, D. J.; Nieto, P. S.; Garbarino-Pico, E.; Avalle, L. B.; Díaz-Fajreldines, H.; Schrurer, C.; Cheng, K.; Guido, M. E. A Non-Mammalian Vertebrate Model of Blindness Reveals Functional Photoreceptors in the Inner Retina. The FASEB J, 2009, 23, 4, 1186-1195.

[18] Valdez, D.; Nieto, P.; Diaz, N.; Garbarino-Pico, E.; Guido, M. E. Differential Regulation of Feeding Rhythms through a Multiple-Photoreceptor System in an Avian Model of Blindness. The FASEB Journal, 2013, 27, 7, $2702-12$.

[19] Valdez, D.J.; Nieto, P.S.; Della Costa, N.S.; Schurrer, C.; Guido M. E. Circadian control of the pupillary light responses in an avian model of blindness, the GUCY1* chickens. Invest Ophthalmol Vis Sci. 2015, 56, 2, 730-737.

[20] Verra, D. M.; Contin, M. A.; Hicks, D.; Guido, M. E. Early onset and differential temporospatial expression of melanopsin isoforms in the developing chicken retina. Invest. Ophthalmol. Vis. Sci. 2011, 52, 5111-5120.

\section{Bio}

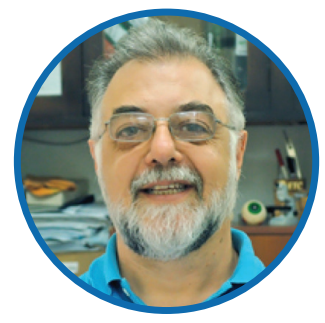

\section{Mario E. Guido}

Mario E. Guido is a full professor at the Department of Biological Chemistry "Ranwel Caputto" (DQBRC), School of Chemistry, National University of Córdoba (UNC), Argentina and Principal Research Investigator of CONICET in the Centro de Investigaciones en Química
Biológica de Cordoba (CIQUIBIC)-CONICET. He did a $\mathrm{PhD}$ in neurochemistry at the UNC (1986-91) and a postdoctoral training in chronobiology at Dalhousie University, Halifax, Canada (1993-97). He runs a laboratory dedicated to investigate the molecular basis of circadian rhythms in lipid metabolism in the nervous system and nonvisual phototransduction mechanisms in the retina. He is vice director of CIQUIBIC, member of the Latin America Academy of Sciences, and JS Guggenheim Memorial Foundation fellow (2009). 